

\section{Los jóvenes: mujeres y hombres, excluidos de las oportunidades de educación y trabajo en Honduras. Una mirada exploratoria acerca de las percepciones sociales sobre los NINI.}

José ACEVEDO*

RESUMEN. Se presenta una mirada exploratoria sobre el fenómeno de los jóvenes NINI en Honduras, basada en la revisión de bibliografía básica acerca del tema y datos estadísticos de fuentes secundarias. Un elemento central del argumento es que la comprensión del fenómeno NINI requiere una clara conceptualización acerca de la juventud y las juventudes. El fenómeno NINI está ligado con la desigualdad social, porque se trata de jóvenes en quienes se combina una doble exclusión, del trabajo y la educación. Los NINI no son nuevos, pero sí relativamente desconocidos en Honduras. El estudio de este fenómeno es clave para visibilizarlos y para el diseño e implementación de políticas, programas y proyectos enfocados en sus necesidades.

Palabras Claves: jóvenes NINI, juventudes, exclusión.

ABSTRACT. An exploratory look at the phenomenon of young NEET in Honduras based on a review of basic literature on the subject and statistical data from secondary sources is presented. Central to the argument is that the understanding of the NEET phenomenon requires a clear conceptualization about youth and youths. Also, young NEET phenomenon is linked to social inequality because they are young who combine a double exclusion, from work and education. The NEET are not new, but relatively unknown in Honduras. The study of this phenomenon is key to get them visible and designing and implementing policies, programs and projects focused on their necessities.

Keywords: young NEET, Youths, exclusion.

\section{Introducción}

El término NINI designa a los jóvenes que ni estudian ni trabajan. Este término se originó en Inglaterra para designar a jóvenes de 16 a 18 años en esa condición, extendiéndose su uso al resto del mundo. Actualmente, las estadísticas en Europa utilizan el rango de 15 a 29 años para calcular los porcentajes de jóvenes que se ubican en la condición de NINI. En Suecia, por ejemplo, la población afectada es el 7.8\%, en Holanda del 5.5\%, mientras que en Bulgaria e Italia, los porcentajes ascienden al $24.6 \%$ y $22.7 \%$, respectivamente (Change, 2014).

Los jóvenes que no estudian ni trabajan constituyen un grupo social extremadamente vulnerable y, por tanto, afectado por la pobreza e inequidad. Afrontan riesgos y desafíos debido a la dificultad que tienen de acceder a oportunidades de educación y al trabajo, especialmente en los ámbitos de seguridad y respeto a los derechos económicos y sociales.

Las investigaciones sobre la magnitud y características de este grupo social deberían ser un paso previo para la formulación de políticas, programas y proyectos enfocados en esa población juvenil.

\section{Metodología}

El presente trabajo se enfoca en responder la siguiente pregunta: ¿Cuál es la magnitud del fenómeno NINI 
en Honduras, su contexto en América Latina y el marco conceptual para su estudio?

Dado que se trata de una mirada exploratoria, cuyo objetivo es situar el tema en el imaginario de los profesionales de las ciencias sociales de Honduras, es fundamental la revisión de la literatura general sobre juventud y, la específica, sobre los NINI.

El primer paso consiste en el análisis del concepto juventud. Ese análisis permitirá identificar los diferentes significados que se le atribuyen al concepto, evidencia de su complejidad y, por tanto, de la imposibilidad de identificarlo con un grupo de edad. Es preciso observar que la existencia de diferentes significados constituye, también, una muestra de las diferentes perspectivas acerca de la juventud.

En segundo lugar, es preciso poner en discusión el concepto mismo de juventud, frente al de juventudes. Es necesario porque si bien es posible identificar algunos rasgos comunes entre los diferentes grupos de jóvenes, las condiciones económicas, sociales y culturales, como el género, la educación o el estado civil, moldean la vida de los jóvenes en cada sociedad, generando profundas diferencias entre grupos de jóvenes. Esta diferenciación justifica la adopción del concepto juventudes, en lugar, del concepto juventud.

En tercer lugar, hay que someter a análisis el concepto NINI, teniendo en cuenta su origen y sus relaciones con la exclusión de oportunidades de educación y trabajo.

Para completar la exploración del tema es imprescindible una revisión de algunas cifras acerca del fenómeno de los NINI, tanto a nivel de Europa como de América Latina y Honduras. En los dos últimos casos, las fuentes son la Organización Internacional del Trabajo (OIT), Comisión Económica para América Latina (CEPAL) y el Instituto Nacional de Estadísticas (INE), de Honduras. La revisión de las cifras muestra el distinto abordaje etario de las instituciones. Mientras la CEPAL y la OIT, se enfocan en la población de 15 a 24 años, el INE lo hace en la población de 12 a 30 años.

En el análisis de las cifras disponibles en las fuentes mencionadas, hay por los menos dos variables cuyo aná- lisis es muy importante. La primera de ellas es la zona de residencia, que permite ver las diferencias entre zona rural y urbana. La segunda es el sexo, porque las variaciones del fenómeno entre hombres y mujeres, constituyen una muestra de las relaciones subyacente de género.

En resumen, el estudio de los NINI permite ampliar la comprensión de la desigualdad social, mediante el análisis de los jóvenes que padecen la doble exclusión de educación y trabajo.

\section{Marco teórico}

Como punto de partida es necesario precisar qué se entiende por esa etapa de la vida de las personas, mujeres y hombres, llamada juventud. La juventud se puede definir de múltiples maneras:

- Como una etapa de la vida. Apuntando que "Dicha definición tiene al menos dos acepciones, por una parte sería una etapa distinguible de otras que se viven en el ciclo de vida humano, como la infancia, la adultez, la vejez; y por otra, es planteada como una etapa de preparación de las y los individuos para ingresar al mundo adulto".

- Como un grupo social definido a partir de la edad. Esta variable “(...) permitiría construir un grupo dentro de las sociedades, a los que se denomina los (y las) jóvenes. En esta versión, se tiende a confundir lo netamente demográfico, un grupo de cierta edad en una sociedad, con un fenómeno sociocultural que es lo juvenil como momento de la vida o como actitud de vida, (...)."

- Como un conjunto de actitudes ante la vida. Donde, "Por ejemplo, se habla de la juventud para decir un estado mental y de salud vital y alegre; se usa también para referirse a un espíritu emprendedor y jovial; también se recurre a ello para hablar de lo que tiene porvenir y futuro; en otras ocasiones se le utiliza para designar aquello que es novedoso y actual, lo moderno es joven (...) Dichas actitudes son mayormente definidas desde el mundo adulto, a partir de una matriz adultocéntrica de comprender y comprenderse en el 
mundo y en las relaciones sociales que en él se dan. Visto así, el mundo adulto se concibe a sí mismo y es visto por su entorno como las y los responsables de formar y preparar a las «generaciones futuras» para su adecuado desempeño de funciones en el mundo adulto, vale decir: como trabajadores, ciudadanos, jefes de familia, consumidores, (...)".

- Como la generación futura. Serán “(...) aquellos y aquellas que más adelante asumirán los roles adultos que la sociedad necesita para continuar su reproducción sin fin. Esta versión tiende a instalar preferentemente los aspectos normativos esperados de las y los jóvenes en tanto individuos en preparación para el futuro (...), sus modos contraculturales de generar agrupamientos, de relacionarse, de comunicarse, de vestirse (...) son vistos como acciones de rebeldía y de no-adaptación social, en tanto no dan cuenta de lo que se espera: la subordinación de las y los jóvenes a lo que cada generación adulta les ofrece en el tiempo que les corresponde vivir. He aquí una franca lucha de poder, entre quienes ofrecen modelos a los cuales adaptarse y quienes intentan producirse y reproducirse desde parámetros propios que las más de las veces contradicen la oferta mencionada" (Duarte Quapper, 2000: 61-66).

Esas distintas concepciones acerca de la juventud, muestran que se trata de una compleja categoría social. Aunque a simple vista la juventud parece ser meramente una categoría natural, asociada a la edad, se trata de una categoría social y, por tanto, sujeta a cambios como resultado del devenir histórico. En otros términos, “(...) se puede afirmar que cuando se hace referencia a la juventud se alude a una condición social con cualidades específicas que se manifiestan, de diferentes maneras, según la época histórica y la sociedad específicamente analizada en cada época" (Villa Sepúlveda, 2011:148).

Hay que agregar que no sólo cambia a lo largo de la historia y sociedades específicas, sino también, en cada sociedad. Es decir, no existe una sola juventud.
Aunque el concepto juventud se usa con frecuencia, algunos autores plantean que es preferible hablar de juventudes debido a que los jóvenes tienen características diferentes dependiendo de factores económicos, sociales o culturales. Por ejemplo, algunos autores plantean que “(...) en la sociedad contemporánea, la noción de juventud se resiste a ser conceptualizada partiendo únicamente de la edad, a ser reducida a mera categoría estadística. De hecho, no hay "juventud", sino juventudes. Se trata de una concepción históricamente construida y determinada, cuya caracterización depende de diferentes variables: la diferenciación social, el género y la generación son las más notorias" (Katoki \& Merli, 2010:14).

La juventud, entendida simplemente como un grupo de edad, tiende a pensarse solo como un período en la vida de las personas. Período durante el cual se pueden dedicar al desarrollo de sus capacidades y a conectarse con el mundo laboral. Esta visión de la juventud no es aplicable a todos los jóvenes, ya que si bien es cierto, los jóvenes que provienen de familias de ingresos altos y medios pueden ajustarse a la misma, los jóvenes pobres de las zonas urbanas y rurales tienen condiciones de vida que no son compatibles con esa imagen (Posas, 2011:7).

Por ejemplo, los niños y niñas que se incorporan al mundo del trabajo, abandonan prematuramente el sistema educativo y terminan enfrentando los desafíos de la vida adulta sin las capacidades que éste suministra y, además, con unas oportunidades limitadas para incorporarse al mundo laboral.

Las adolescentes que resultan embarazadas se incorporan de una manera rápida y brutal al mundo adulto. Lo hacen sin la oportunidad de desarrollar suficientes destrezas laborales y, además, con muy limitadas posibilidades de trabajar y, muchas, de reproducir y feminizar la pobreza.

Aunque existen muchas otras fuentes, estos casos representan ejemplos clave para entender la existencia de vastos grupos de jóvenes excluidos de la educación y el trabajo. 
3.1 Los NINI, mujeres y hombres, que ni estudian ni trabajan

Los jóvenes que ni estudian ni trabajan no son un fenómeno reciente, pero sí constituyen una preocupación que se viene expresando con mayor fuerza hace poco tiempo, porque ya resulta evidente que este “...fenómeno pone de manifiesto los altos costos sociales de la marginación de los jóvenes dos de los principales mecanismos de integración social, la escuela y el trabajo, observándose el problema como una cuestión de exclusión social, falta de oportunidades de los jóvenes y abandono del Estado". (OIT. Oficina Regional para América Latina y el Caribe, 2013:72-73).

Así como se debe hablar de juventudes, también, se debería hablar de diversas juventudes NINI. La razón por la que se les aborda como una unidad es porque hay una característica común “(...) que los ubica en una misma categoría es precisamente el permanecer al margen de algunas de las instituciones clave de socialización e integración social durante esa etapa de la vida. Tal condición de exclusión los coloca en una situación de indefinición cercana a la inexistencia social, por cuanto la sociedad contemporánea no logra asignarles un reconocimiento y/o estatus social particular: No son estudiantes, trabajadores, desocupados, ni amas de casa. Esa situación extrema de exclusión, en términos de desafiliación social, convierte a este grupo no solo en manifestación evidente de la crisis por la que atraviesan los canales tradicionales de integración social, sino al mismo tiempo en una caja negra de la que poco sabemos, pero que fácilmente se asocia con diversos e incluso contrastantes prejuicios sociales: desde una prolongada adolescencia que se resiste a asumir responsabilidades adultas, hasta la estigmatización de este grupo con actividades delictivas, consumo de drogas y otras prácticas socialmente sancionadas" (Saraví, 2004:71).

\subsection{Las percepciones}

La identificación y análisis de los riesgos y desafíos de los jóvenes, mujeres y hombres, sin acceso a educación y trabajo, a través del estudio de una doble perspectiva, la de los adultos y, la de los propios jóvenes, requiere una investigación rigurosa de las percepciones de ambos grupos. En el caso de los adultos y las instituciones, generalmente dirigidas por los adultos, se trata de auscultar sus actitudes y concepciones frente a los jóvenes y, particularmente, los NINI.

En el caso de los jóvenes, el estudio deberá de enfocarse en las percepciones que los jóvenes tienen de sí mismos y de su propia situación. Así como sobre las políticas públicas elaboradas, generalmente, por adultos, para mejorar su situación.

En el análisis de esas percepciones habrá que tener en cuenta la relación de poder existente entre juventud y adultez, lo que se expresa en la subordinación de los jóvenes a los adultos. Según Villa Sepúlveda, "Lo que sueñan que debe ser lo social tiende a ser distinto a las concepciones y a las prácticas adultas. En consonancia, las y los jóvenes construyen concepciones sociales, teorías sociales, imaginarios sociales y utopías que prescriben sus prácticas por las que es necesario indagar para entender los sentidos por los que van transitando las sociedades occidentales y occidentalizadas, que tienden, en la actualidad, a la juvenilización y a la infantilización de la cultura. Es decir, a asumir como legítimos los sentidos que producen las nuevas generaciones, en tanto éstos sean simétricos a lógica mercantil en la que, paulatinamente, se van sumergiendo" (Et al, 2011:156).

Parafraseando a esta autora, se puede afirmar que los adultos tienen sus propias concepciones e imaginarios sobre lo que deberían ser las prácticas de los jóvenes y, más aún, sobre los riesgos que representan las prácticas colectivas de los jóvenes.

El estudio de las percepciones deberá hacerse a través de la identificación y análisis de las representaciones sociales de ambos grupos, las cuales adquieren una gran importancia porque orientan las prácticas colectivas, tanto de jóvenes como de adultos.

En este estudio, la identificación de varias juventudes cobra importancia porque cada una de ellas puede tener diferentes representaciones ${ }^{1}$. Al respecto, Klaudio Duarte, plantea que "Cuando en nuestras sociedades 
se habla de la juventud, se está haciendo referencia a varios sentidos simultáneamente. La necesaria contextualización de los discursos y acciones al respecto, y el reconocimiento de la posición de quien habla, permitirá una mejor comprensión de estos discursos. Una primera idea fuerza de esta reflexión es que la nominación en singular de la juventud no es tal, ya que sus significantes son diversos y refieren a varias imágenes desde un mismo habla con diversos hablantes. ¿Cuáles son esos diversos significados o usos que se hace de la categoría juventud?" (Et al, 2000:61).

\section{Análisis preliminar del problema}

En un estudio para 18 países latinoamericanos, que incluye los cinco países centroamericanos, la OIT encontró que, en 2011, el 20.3\% de los jóvenes de 15 a 24 años eran NINI, es decir no trabajaban ni estudiaban. Las cifras, también, indican la existencia de una importante brecha de género entre ellos, ya que el 12.0\% eran hombres y, el 28.6\%, mujeres.

En Honduras esta situación es particularmente grave. De acuerdo con los datos de la Encuesta de Hogares de Mayo del 2013 (publicados por el INE), se señala que los jóvenes de entre 12 y 30 años de edad, ascienden a 3,302,206, representando el $43.2 \%$ de la población hondureña. De éstos, 1,630,291 son hombres (49.4\%) y, 1,671,916 (50.6\%), son mujeres. Del total de jóvenes, un $26.4 \%$ que equivale a aproximadamente 872,994 personas, ni estudia ni trabaja, lo que les ubica en la categoría de NINI.

Las cifras de la CEPAL referidas únicamente a la población de 15 a 24 años, por su parte, muestran que el fenómeno ha experimentado pocas variaciones cuantitativas en el período 2001-2010, ya que apenas pasó de $26.3 \%$ a $25 \%$. En el grupo de 20 a 24 años, con un mayor porcentaje de población NINI, la variación fue mínima puesto que en el 2001 alcanzó el 30.7\%, disminuyendo a 29.1\%, en el 2010. Solamente en el grupo de 15 a 19 años hubo una disminución de mayor magnitud, considerando que en el 2001 alcanzó el 26.3\%, llegando al 21.8\%, en el 2010 (Gráfico 1).

\section{Gráfico 1. Honduras: jóvenes que no estudian ni trabajan}

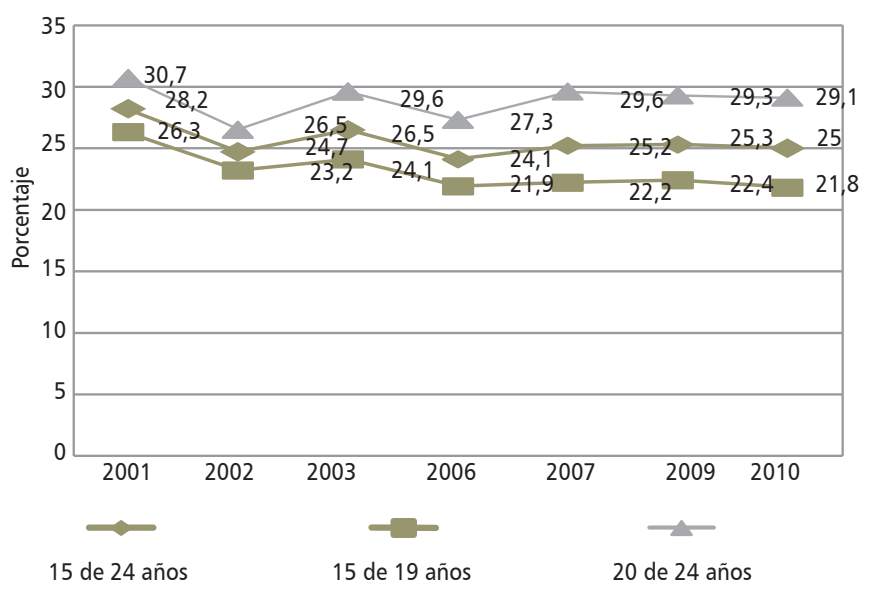

Fuente: elaboración propia con datos de http://interwp.cepal.org/sisgen/ConsultalntegradaFlashProc_HTML.asp

Las disparidades de género en materia laboral son significativas. Entre la población juvenil que trabaja, el $69.3 \%$ son hombres y, apenas, el 30.7\% lo constituyen mujeres. Esta disparidad se profundiza al analizar el caso de los NINI, donde el $80.3 \%$ son mujeres y, solamente, el 19.7\% son hombres (INE, EPHPM, 2013).

Un dato a destacar entre la población NINI es en términos de concentración geográfica. Los jóvenes que no estudian y tampoco trabajan, son más en la zona rural que en la urbana. Eso hace que el fenómeno NINI sea más rural que urbano, lo que probablemente está relacionado con el menor acceso a educación en el área rural.

Los NINI están lejos de ser un grupo homogéneo. Sobre este particular, un estudio de la OIT precisa que es “(...) necesario señalar que los NINI son un grupo muy heterogéneo, conformado por realidades muy disímiles.

1. Aunque en esta propuesta se plantea la existencia de los NINI, es muy probable que haya que realizar importantes distinciones. Por ejemplo, entre, NINI urbano y rural, o, entre mujeres y hombres NINI. 
Se incluyen quienes no trabajan ni estudian pero buscan empleo, a los jóvenes dedicados a los quehaceres de hogar (en su mayoría mujeres) y, a otros inactivos (principalmente hombres), que no trabajan, ni estudian, no buscan empleo ni realizan tareas del hogar. Este último es un "núcleo duro", sin actividad específica, muchos de los cuales se encuentran transitoriamente en esta situación, o en una transición entre diferentes actividades laborales o educativas". (OIT, 2013)

Precisamente, debido a esta heterogeneidad, la identificación de las causas resulta un ejercicio complejo. La exclusión del sistema educativo se puede atribuir, de entrada, a la situación de pobreza de las familias de las cuales provienen. En tanto, que la exclusión del mundo laboral se origina, parcialmente, por el escaso dinamismo del sistema económico que no genera suficientes empleos para la población juvenil que se incorpora anualmente al mercado de trabajo.

La disparidad entre las cifras de NINI mujeres y NINI hombres, parece indicar, también, que la desigualdad de género que prevalece en Honduras profundiza la exclusión de las mujeres del mundo laboral y educativo.

Debido a las disparidades de género, las jóvenes NINI representan el 21.2\% de la población total femenina de Honduras. Con, alrededor, de 701,007 mujeres jóvenes excluidas de la educación y el empleo. En tanto, los hombres jóvenes NINI, representan el 5.2\% de la población masculina total, es decir, unos 171,987 jóvenes (INE, 2013).

La realización de estudios sobre los jóvenes resulta de considerable importancia. Especialmente en países, que como Honduras, presentan elevados niveles de exclusión, debido a que esta categoría social es muy vulnerable. Por ejemplo, "el ingreso promedio nacional que percibe esta población incorporada en el mercado laboral es de 4,574 .00 Lempiras mensuales, en el área urbana y rural se estiman en 5,644.00 Lempiras y 3,165.00 Lempiras respectivamente" (INE, 2013:9). Entre ellos, los NINI se encuentran en situación de mayor vulnerabilidad, ya que la misma definición supone que ellos están excluidos de la educación y el trabajo, lo que significa que carecen de ingresos propios.

\section{Las percepciones acerca de los NINI}

Varias son las preguntas que sobre este grupo, NINI, se pueden formular: ¿Cuáles son las percepciones que tienen los actores relevantes sobre los riesgos que enfrentan los jóvenes excluidos del mundo de la educación y el trabajo? ¿Cómo enfrentar el estigma colectivo de que los NINI representan un riesgo por violencia y consumo de drogas? ¿Cómo entender el impacto que tiene la exclusión sobre la imagen de sí mismos, acerca de su presente y su futuro?

De igual forma, se deben identificar los desafíos desde su propia perspectiva, pero, también, desde la visión del resto de la sociedad. Esto implica conocer las percepciones acerca del impacto de las políticas públicas enfocadas, directa o indirectamente, en este sector de juventud excluida. Así, este conocimiento puede ser un aporte para diseñar nuevas orientaciones para la formulación de políticas públicas que contribuirán a la reducción de la exclusión y sus consecuencias.

En todo caso, los NINI hombres y mujeres, especialmente urbanos, son percibidos como un grupos en riesgos y riesgosos en el contexto de la vulnerabilidad juvenil. No obstante, el conocimiento que existe en Honduras sobre los diferentes tipos de NINI, según los factores causantes de su exclusión en términos de educación y empleo, es notoriamente insuficiente. También, es escaso el conocimiento sobre las percepciones que tienen los diferentes sectores sociales acerca de estos jóvenes. Más escaso aún es el conocimiento generado acerca de las percepciones que tienen los jóvenes sobre los desafíos que enfrentan debido a la exclusión.

Es preciso anotar, también, que los jóvenes que viven en situación de pobreza y, particularmente los NINI, son percibidos como una fuente de riesgo y, lo que es aún peor, se perciben a sí mismos como personas sin futuro. Esta auto-percepción está basada en el nulo o insuficiente acceso a formación en habilidades para la vida.

La situación de los jóvenes en Honduras se complica mucho más debido a la situación de violencia generali- 
zada que hay en el país y que le posiciona entre los más violentos del mundo. Eso hace que los jóvenes en Honduras constituyan uno de los grupos con mayor riesgo de muerte por violencia homicida. Por ejemplo, según datos del Observatorio de la Violencia del IUDPAS, el 44\% de los homicidios del 2013, tuvieron como víctima a un joven de entre 15 y 29 años.

De igual manera, en ese mismo año, el 48\% de los suicidios ocurrió en ese mismo grupo de edades. Considerando su elevado nivel de vulnerabilidad, es muy probable que una cantidad importante de homicidios y suicidios hayan tenido a un NINI como víctima.

En los últimos años la situación de inseguridad que enfrentan los jóvenes ha elevado la tasa de migración juvenil. Lo que significa que Honduras está perdiendo la oportunidad de aprovechar su bono demográfico. Se pierde al excluir a jóvenes del mundo educativo y laboral. Igual se pierde como resultado de la violencia criminal y los suicidios y, también, se pierde debido a la migración por la inseguridad.

Es preciso señalar que aunque en los últimos años han sido aprobadas políticas públicas, a nivel nacional y municipal, enfocadas en los jóvenes, no existen políticas específicas para los NINI, lo que indica que este grupo de jóvenes se ha mantenido en la invisibilidad.

\section{La respuesta desde las políticas socia- les}

Hay que reconocer, como ya fue apuntado, que aunque el fenómeno de los NINI no es nuevo, es relativamente desconocido en el país. Explorar este fenómeno desde una perspectiva de investigación no sólo sirve para informar a los tomadores de decisiones, sino también, para sensibilizar a la población, creando así un ambiente más favorable para el diseño e implementación de políticas, programas y proyectos enfocados en los jóvenes que presentan mayores niveles de vulnerabilidad: los NINI.

Las políticas públicas elaboradas a favor de los jóvenes son: la Política Pública Nacional de la Juventud y, la Política Nacional de Prevención de Violencia hacia la Niñez y Juventud en Honduras. Además de estas políticas, existen políticas transversales que pueden contribuir a mejorar la calidad de vida de los jóvenes, entre las que se cuentan: la Política de Protección Social, la Política Pública en Derechos Humanos y, la Política Nacional de la Mujer, Primer y Segundo Plan Nacional.

Es preciso reconocer que a nivel municipal (aunque sólo en algunos municipios), se están realizando acciones para combatir la problemática a través de políticas municipales de juventud. Estas medidas sirven como instrumento para guiar la gestión e inversión de las municipalidades para satisfacer las necesidades y resolver problemas de sus comunidades.

No obstante, la eficacia de las políticas es muy limitada debido a la carencia de un enfoque integral para la ejecución de las políticas públicas, a nivel nacional y municipal. Esto resulta en esfuerzos aislados para responder al incumplimiento de los derechos de los jóvenes. Aunque la legislación nacional y su institucionalidad parecen estar encaminadas a la protección social, carecen de un enfoque integral, de modo que no aportan los insumos necesarios para su adecuada ejecución a nivel nacional y local.

\section{Reflexión final}

En síntesis, los elevados niveles de exclusión social que caracterizan a Honduras, combinados con la poca eficacia de las políticas de juventud, dan lugar a fenómenos como los NINI.

A pesar de que hay algunos estudios sobre la situación de la juventud en Honduras, los estudios sobre los NINI son inexistentes. De hecho, no es sino hasta en el año 2001, que el término comenzó a ser utilizados en las publicaciones y/o estadísticas oficiales.

La falta de estudios que incluyan las perspectivas de los jóvenes y los adultos sobre la población NINI y que, además, contribuyan a su caracterización, constituye un importante desafío para las ciencias sociales y los investigadores nacionales. 


\section{Bibliografía}

- Change, E. m. (9 de Abril de 2014). Young people and 'NEETs'. Obtenido de http://www.eurofound. europa.eu: http://www.eurofound.europa.eu/emcc/ labourmarket/youth.htm

- Duarte Quapper, K. (2000). iJuventud o juventudes? Acerca de como mirar y remirar a las juventudes de nuestro continente. Ultima década №13, 59-77.

- Duarte Quapper, K. (2000). Duarte Quapper, Klaudio. ¿Juventud o juventudes? Acerca de cómo mirar y remirar a las juventudes en nuestro continente. 2000. Ultima Década. No. 13. Recuperado el 25 de Agosto de 2014, de http://www.captura.uchile.cl/bitstream/ handle/2250/131803/Juventud_o_juventudes_versiones.pdf? sequence $=1$

- INE. (2013). Resumen ejecutivo. XLIV encuesta de hogares de propósitos múltiples Mayo 2013. INE, Tegucigalpa.
- IUDPAS, (2001) Observatorio de la Violencia, Boletín.

- Katoki, M., \& Merli, L. (2010). Las representaciones sociales que construyen diferentes jóvenes sobre las mujeres. En F. d. UNLP (Ed.), XIV jornadas nacionales de investigadores en comunicación.

- OIT. Oficina Regional para América Latina y el Caribe. (2013). Panorama Laboral 2013. América Latina y el Caribe. OIT, Lima.

- Posas, M. (2011). Una mirada sobre y desde la juventud: grupos focales de jóvenes y adultos. Tegucigalpa.

- Saraví, G. A. (Enero - febrero de 2004). Entre la evasión y la exclusión social: jóvenes que no estudian ni trabajan. Una exploración del caso argentino. Nueva Sociedad(189), 69-84.

- Villa Sepúlveda, M. E. (Mayo - agosto de 2011). Del concepto de juventud al de juventudes y al de lo juvenil. Revista Educación y Pedagogía, 147 - 157. 